\title{
BOUND STATES IN WEAKLY DEFORMED WAVEGUIDES: NUMERICAL VERSUS ANALYTICAL RESULTS
}

\author{
PAOLO AMORE ${ }^{\varpi 1}$, JOHN P. BOYD ${ }^{2}$, FRANCISCO M. FERNÁNDEZ ${ }^{3}$, \\ MARTIN JACOBO $^{4}$ and PETR ZHEVANDROV 5
}

(Received 3 July, 2016; accepted 22 October, 2016)

\begin{abstract}
We study bound states in weakly deformed and heterogeneous waveguides, and compare analytical predictions using a recently developed perturbative method with precise numerical results for three different configurations: a homogeneous asymmetric waveguide, a heterogenous asymmetric waveguide and a homogeneous broken strip. We have found excellent agreement between the analytical and numerical results in all the examples; this provides a numerical verification of the analytical approach.
\end{abstract}

2010 Mathematics subject classification: primary 81Q15; secondary 35P15, 65N25.

Keywords and phrases: trapped modes, bound states, deformed waveguide.

\section{Introduction}

The appearance of trapped modes (bound states) in open geometries under perturbations has attracted much attention in both physical and mathematical literature recently (see, for example, the books by Exner and Kovařík [12], Hurt, [15] and Londergan et al. [17]). A classical example is the appearance of a bound state for the one-dimensional Schrödinger operator perturbed by a small potential well [21]. In this situation, the unperturbed problem possesses a purely continuous spectrum corresponding to plane waves $\exp (i k x)$ with energy $E=k^{2}$, so that the continuous spectrum occupies the positive ray $0 \leq E<\infty$. Under a perturbation by a potential well $\epsilon V(x)$ with $\epsilon \rightarrow 0^{+}$and a smooth and compactly supported function $V$, such that $\int V(x) d x<0$, the threshold $E=0$ yields a bound state, whose energy $E_{\epsilon}$ is located

\footnotetext{
${ }^{1}$ Facultad de Ciencias, CUICBAS, Universidad de Colima, Bernal Díaz del Castillo 340, Colima, Colima, Mexico; e-mail: paolo.amore@gmail.com.

${ }^{2}$ Department of Atmospheric, Oceanic \& Space Science, University of Michigan, 2455 Hayward Avenue, Ann Arbor, MI 48109, USA; e-mail: jpboyd@umich.edu. ${ }^{3}$ INIFTA (UNLP, CCT La Plata-CONICET), Division Quimica Teorica, Blvd. 113 S/N, Sucursal 4, Casilla de Correo 16, 1900 La Plata, Argentina; e-mail: fernande@quimica.unlp.edu.ar. ${ }^{4}$ Facultad de Ciencias, Universidad de Colima, Bernal Díaz del Castillo 340, Colima, Colima, Mexico. ${ }^{5}$ Facultad de Ciencias Físico-Matemáticas, Universidad Michoacana de San Nicolás de Hidalgo, Ciudad Universitaria, 58030 Morelia, Michoacán, Mexico; e-mail: pzhevand@ gmail.com. (C) Australian Mathematical Society 2017, Serial-fee code 1446-1811/2017 \$16.00
} 
to the left of the continuous spectrum close to the threshold, $E_{\epsilon}=-c \epsilon^{2}$. Here, the constant $c$ is proportional to the square of the area above the graph of $V(x)$ (see Simon's paper [21] for details).

This mechanism of the generation of bound states by the threshold of the continuous spectrum seems to be quite generic, and is analogous to many situations of physical interest [12, 15, 17]. For example, Bulla et al. [8] discovered that a similar phenomenon occurred in a slightly deformed waveguide described by the Laplace operator with Dirichlet boundary conditions on the walls. They showed that, under certain perturbations (which enlarge the waveguide), the threshold of the continuous spectrum gave rise to an eigenvalue to the left of it. The distance of this eigenvalue from the continuous spectrum is analytic in the perturbation parameter, when the latter is sufficiently small. Bulla et al. [8] also gave an explicit formula for the leading term in the expansion of the eigenvalue in the Taylor series with respect to this parameter. They used the so-called Birman-Schwinger technique [19] to obtain these results. Subsequently, many other approaches to problems of this kind were developed (see, for example, the works of Borisov et al. [6], Exner and Kovařík [12] and Nazarov [18]). For the most part, these techniques provide approximate formulae for the eigenvalues up to a certain power of the perturbative parameter, but it is impossible to say whether the formulae approximate the eigenvalues for a given fixed value of the parameter. A goal of this paper is the investigation of this question for several examples from waveguide theory by comparing precise numerical results, obtained by means of the collocation method, with variational estimates and the first nonvanishing terms provided by the theoretical formulae of different perturbative approaches.

The principal difficulty of the problems under consideration is that in each case the nonperturbed problem does not have eigenvalues, so that the standard regular perturbation theory is not applicable. Recently, yet another perturbative approach, which extends a method previously developed by Gat and Rosenstein [13], was proposed by Amore et al. [1, 2]. It has the advantage of using an auxiliary "unperturbed" problem, which does possess an eigenvalue and is exactly solvable, so that a standard perturbation procedure can be used to construct corrections up to any order. A comparison of previously known theoretical results, as well as precise numerical calculations, with this new perturbative approach constitutes the second goal of this paper.

Note that the approach of Amore et al. [1,2] is equally applicable to weakly deformed or weakly heterogeneous waveguides. Therefore, we have chosen the following three examples: (a) an asymmetrically deformed waveguide (this is exactly the case considered by Bulla et al. [8]); (b) an asymmetrically deformed waveguide with a localized heterogeneity (we are not aware of the existence of any results for this case in the literature); (c) a broken strip (investigated by different methods of Avishai et al. [4] and Granot [14]).

The paper is organized as follows. In Section 2, we summarize the results of Amore et al. [1,2]. In Section 3, we present a comparison of the results of different approaches to the three examples mentioned. We draw some conclusions in Section 4. 


\section{Method}

The problem of calculating the emergence of trapped states in infinite slightly heterogeneous waveguides was recently considered by Amore et al. [1, 2], who obtained exact perturbative formulae that use the density inhomogeneity as a perturbation parameter. This approach extends a method previously developed by Gat and Rosenstein [13] for calculating the binding energy of threshold bound states.

In particular, Amore et al. [2] gave a calculation up to third order, while Amore [1] extended the calculation to fourth order. These formulae have been tested on two exactly solvable models, reproducing the exact results up to fourth order.

Although the examples considered by Amore et al. [1, 2] were limited to the case of heterogeneous straight waveguides, the perturbative expressions apply to the case of homogeneous and slightly deformed waveguides, and to the more general case of slightly heterogeneous and slightly deformed waveguides. This paper focuses on these last two cases. Incidentally, while the effect of small deformations on infinite and homogeneous waveguides has been studied before by different authors using different techniques (see, for example, the book by Exner and Kovařík [12] and the references therein), the effect of weak heterogeneities on infinite (either straight or deformed) waveguides is much less well known. In this paper, we compare the theoretical predictions of the formulae obtained by Amore et al. [1,2] with numerically precise results for different models.

We refer the reader to the perturbative expansion of Amore et al. [1, 2], and here we give only the general formulae for the perturbative corrections to the energy of the fundamental mode of a heterogeneous waveguide up to fourth order, as follows:

$$
\begin{aligned}
& E_{0}^{(1)}=0, \\
& E_{0}^{(2)}=-\frac{\pi^{2}}{b^{2}} \Delta_{2}^{2}, \\
& E_{0}^{(3)}=-2 \frac{\pi^{2}}{b^{2}} \Delta_{2}\left(\Lambda_{1}-\Delta_{3}\right), \\
& E_{0}^{(4)}=-\frac{\pi^{2}}{b^{2}}\left[-2 \Delta_{2}^{4}-\Delta_{2}^{2} \Delta_{4}+2 \Delta_{2} \Delta_{5}+\Delta_{3}^{2}-2 \Lambda_{2}-\Delta_{3} \Lambda_{1}+2 \Delta_{2} \Lambda_{3}+\Lambda_{1}^{2}\right] .
\end{aligned}
$$

Here we use the following definitions of Amore [1]:

$$
\begin{aligned}
& \Delta_{1} \equiv \frac{\pi}{b^{2}} \int \sigma(x, y) d x d y, \\
& \Delta_{2} \equiv \frac{\pi}{b^{2}} \int \sigma(x, y) \cos ^{2} \frac{\pi y}{b} d x d y, \\
& \Delta_{3} \equiv \frac{\pi^{3}}{b^{5}} \iint \sigma\left(x_{1}, y_{1}\right) \sigma\left(x_{2}, y_{2}\right)\left|x_{1}-x_{2}\right| \cos ^{2} \frac{\pi y_{1}}{b} \cos ^{2} \frac{\pi y_{2}}{b} d x_{1} d y_{1} d x_{2} d y_{2}, \\
& \Delta_{4} \equiv \frac{\pi^{4}}{b^{6}} \iint \sigma\left(x_{1}, y_{1}\right) \sigma\left(x_{2}, y_{2}\right) x_{1}\left(2 x_{2}-x_{1}\right) \cos ^{2} \frac{\pi y_{1}}{b} \cos ^{2} \frac{\pi y_{2}}{b} d x_{1} d y_{1} d x_{2} d y_{2},
\end{aligned}
$$




$$
\begin{aligned}
\Delta_{5} \equiv & \frac{\pi^{5}}{b^{8}} \iiint \sigma\left(x_{1}, y_{1}\right) \sigma\left(x_{2}, y_{2}\right) \sigma\left(x_{3}, y_{3}\right)\left|x_{1}-x_{2}\right|\left|x_{2}-x_{3}\right| \\
& \times \cos ^{2} \frac{\pi y_{1}}{b} \cos ^{2} \frac{\pi y_{2}}{b} \cos ^{2} \frac{\pi y_{3}}{b} d x_{1} d y_{1} d x_{2} d y_{2} d x_{3} d y_{3}, \\
\Lambda_{1} \equiv & \frac{\pi^{3}}{b^{4}} \iint \sigma\left(x_{1}, y_{1}\right) \sigma\left(x_{2}, y_{2}\right) \cos \frac{\pi y_{1}}{b} \cos \frac{\pi y_{2}}{b} g_{2}^{(0,0)}\left(x_{1}, y_{1}, x_{2}, y_{2}\right) d x_{1} d y_{1} d x_{2} d y_{2}, \\
\Lambda_{2} \equiv & \frac{\pi^{6}}{b^{9}} \iiint \int \sigma\left(x_{1}, y_{1}\right) \sigma\left(x_{2}, y_{2}\right) \sigma\left(x_{3}, y_{3}\right) \sigma\left(x_{4}, y_{4}\right)\left|x_{1}-x_{3}\right| \cos \frac{\pi y_{1}}{b} \cos \frac{\pi y_{2}}{b} \\
& \times \cos ^{2} \frac{\pi y_{3}}{b} \cos ^{2} \frac{\pi y_{4}}{b} g_{2}^{(0,0)}\left(x_{1}, y_{1}, x_{2}, y_{2}\right) d x_{1} d y_{1} d x_{2} d y_{2} d x_{3} d y_{3} d x_{4} d y_{4}, \\
\Lambda_{3} \equiv & \frac{\pi^{5}}{b^{6}} \iiint \sigma\left(x_{1}, y_{1}\right) \sigma\left(x_{2}, y_{2}\right) \sigma\left(x_{3}, y_{3}\right) \cos \frac{\pi y_{1}}{b} \cos \frac{\pi y_{3}}{b} \\
& \times g_{2}^{(0,0)}\left(x_{1}, y_{1}, x_{2}, y_{2}\right) g_{2}^{(0,0)}\left(x_{2}, y_{2}, x_{3}, y_{3}\right) d x_{1} d y_{1} d x_{2} d y_{2} d x_{3} d y_{3} .
\end{aligned}
$$

The correction to the energy of the fundamental mode up to fourth order can be put in the form

$$
\Delta E_{0} \approx E_{0}^{(2)}+E_{0}^{(3)}+E_{0}^{(4)}=-\frac{\pi^{2}}{b^{2}}\left\{\left(\Delta_{2}+\left(\Lambda_{1}-\Delta_{3}\right)^{2}\right)^{2}+\Gamma\right\},
$$

where

$$
\Gamma \equiv\left[-2 \Delta_{2}^{4}+\Delta_{2} \Delta_{3}-\Delta_{2}^{2} \Delta_{4}+2 \Delta_{2} \Delta 5-\Delta_{3} \Lambda_{1}-2 \Lambda_{2}+2 \Delta_{2} \Lambda_{3}\right] .
$$

Equation (2.1) will be applied in the next section to different waveguides.

\section{Application to deformed waveguides}

The perturbative formulae obtained by Amore et al. [1,2] apply to the general case of heterogeneous and deformed waveguides, although the applications considered in those papers were concerned only with slightly heterogeneous straight waveguides.

An appropriate conformal map $w \equiv u+i v=F(z)$ can map an infinite strip, $y \in$ $\left(b_{-}, b_{+}\right)$, with $b_{-}<b_{+}$, and $-\infty<x<\infty$, onto a deformed waveguide, $u \in(-\infty, \infty)$ and $f_{-}(u)<v<f_{+}(u)$. Here $f_{ \pm}(u)$ are the upper and lower borders of the deformed strip, respectively, over which the Dirichlet boundary conditions are assumed.

Suppose that one has to solve the Helmholtz equation for the deformed strip assumed to be heterogeneous, and with a physical density varying at each point, $\rho(u, v)>0$. We also require that the density variations are small and localized around one (or more) points internal to the domain or, equivalently, that $\rho(u, v)$ tends sufficiently rapidly to a constant value $\rho_{0}$, as $|u| \rightarrow \infty$.

One then has to solve the eigenvalue equation

$$
-\Delta_{u, v} \phi(u, v)=E \rho(u, v) \phi(u, v),
$$

with $\phi\left(u, f_{ \pm}(u)\right)=0$, and $\Delta_{u, v} \equiv\left(\partial^{2} / \partial u^{2}\right)+\left(\partial^{2} / \partial v^{2}\right)$. Here, $E$ and $\phi$ are, respectively, the eigenvalues and eigenfunctions of the Helmholtz equation on the original domain. 
If we map the deformed strip back to the straight waveguide, equation (3.1) transforms into

$$
-\Delta_{x, y} \psi(x, y)=E \Sigma(x, y) \rho(u(x, y), v(x, y)) \psi(x, y),
$$

where $\Sigma(x, y)=|d F / d z|^{2}$ (we will refer to it as the "conformal density"), and $\psi\left(x, b_{ \pm}\right)=$ 0 . In this case, $\psi$ are the eigenfunctions of equation (3.2) on the transformed domain. From a physical point of view, equation (3.1) can be interpreted as the Helmholtz equation for a straight waveguide with a physical density

$$
\tilde{\rho}(x, y) \equiv \Sigma(x, y) \rho(u(x, y), v(x, y)) .
$$

Under the assumptions of small deformations and weak heterogeneity,

$$
\tilde{\rho}(x, y)=\rho_{0}+\tilde{\sigma}(x, y),
$$

where $|\tilde{\sigma}(x, y)| \ll 1$, and $\lim _{|x| \rightarrow \infty} \tilde{\sigma}(x, y)=0$. In this case, equation (3.2) has precisely the same form given by Amore et al. [1, 2], and one can straightforwardly apply their perturbative formulae to calculate the corrections to the lowest eigenvalue for a waveguide that is both deformed and heterogeneous. (Finding the conformal map that sends a given deformed waveguide into a straight waveguide may still be a difficult challenge, but we are not concerned with this issue here.)

In the following, we examine three examples: an asymmetric homogeneous waveguide with a local enlargement, an asymmetric heterogeneous waveguide with a local narrowing, and a slightly broken strip with a homogeneous density.

3.1. Asymmetrically deformed waveguide Bulla et al. [8] considered the waveguide on the domain

$$
\Omega_{\lambda}=\left\{(x, y) \in \mathbb{R}^{2} \mid 0<y<1+\lambda f(x)\right\}
$$

and found that the fundamental mode of the Laplacian on this domain, for the Dirichlet boundary conditions at the border, behaves as

$$
E(\lambda)=\pi^{2}-\pi^{4} \lambda^{2}\left(\int_{\mathbb{R}} f(x) d x\right)^{2}+O\left(\lambda^{3}\right) .
$$

Note that $\lambda$ in equation (3.3) is a parameter controlling the deformation (in particular, for $\lambda=0$ one has a straight waveguide).

Since the formulae of Amore et al. [1,2] apply to this domain as well, we consider the conformal map

$$
F(z)=z+a \tanh \left(\frac{z}{z_{0}}\right)
$$

which transforms a straight waveguide of unit width into a waveguide of the kind considered by Bulla et al. [8]. The waveguide obtained using this map for $a=1 / 5$ and $z_{0}=1$ is displayed in Figure 1. Observe that $F(z)$ has simple poles at $z=i(2 k+1) \pi z_{0} / 2$ with $k=0, \pm 1, \pm 2, \ldots$ 


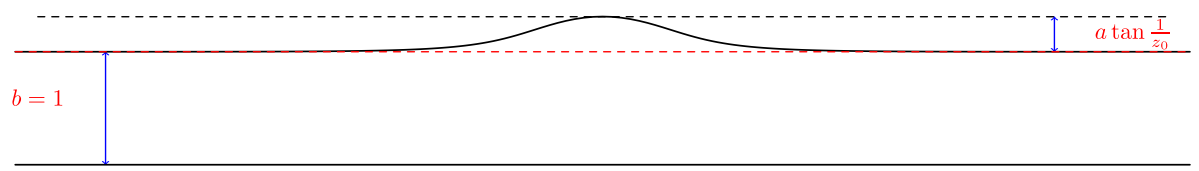

FIGURE 1. Asymmetric waveguide corresponding to the map in equation (3.5). The waveguide is oriented along the $x$-axis. Note that $b$ is the width of the waveguide at $|x| \rightarrow \infty$.

The lower side of the waveguide is not deformed, whereas the upper side is deformed to the parametric curve

$$
\left\{\begin{array}{l}
u(x)=x+\frac{a \sinh \left(2 x / z_{0}\right)}{\cos \left(2 / z_{0}\right)+\cosh \left(2 x / z_{0}\right)} \\
v(x)=1+\frac{a \sin \left(2 / z_{0}\right)}{\cos \left(2 / z_{0}\right)+\cosh \left(2 x / z_{0}\right)}
\end{array}\right.
$$

with $x \in(-\infty, \infty)$. In this case, the conformal density is given by $\Sigma(x, y)=1+\sigma(x, y)$, where

$$
\sigma(x, y)=\frac{4 a\left\{\cosh \left(2 x / z_{0}\right) \cos \left(2 y / z_{0}\right)+1\right\}}{z_{0}\left\{\cosh \left(2 x / z_{0}\right)+\cos \left(2 y / z_{0}\right)\right\}^{2}}+\frac{4 a^{2}}{z_{0}^{2}\left\{\cosh \left(2 x / z_{0}\right)+\cos \left(2 y / z_{0}\right)\right\}^{2}} .
$$

The area corresponding to the enlargement, that is, the area $1<y<1+a \tan 1 / z_{0}$, is obtained by the formula

$$
\begin{aligned}
\delta \mathcal{A} & =\int_{-\infty}^{\infty} f(u) d u=\int_{-\infty}^{\infty} \frac{a \sin \left(2 / z_{0}\right)}{\cosh \left(2 x / z_{0}\right)+\cos \left(2 / z_{0}\right)} d x+O\left(a^{2}\right) \\
& =2 a+O\left(a^{2}\right) .
\end{aligned}
$$

For $\delta \mathcal{A}>0$, one can apply the formula of Bulla et al. [8]; in this case, the lowest eigenvalue of the deformed waveguide

$$
E \approx \pi^{2}-4 \pi^{4} a^{2}+O\left(a^{3}\right)
$$

(Observe that in this case we are not allowed to calculate the enlargement using the formula $\int_{-\infty}^{\infty} d x \int_{0}^{1}(\Sigma(x, y)-1) d y$, since both integrals $\int \Sigma(x, y) d x d y$ and $\int 1 d x d y$ are divergent.)

We now calculate the dominant behaviour of the lowest eigenvalue of the waveguide using the expression for the second-order contribution of Amore et al. [2]; in this case,

$$
\begin{aligned}
\Delta_{2} & =\pi \int_{-\infty}^{\infty} d x \int_{0}^{1} \sigma(x, y) \sin ^{2}(\pi y) d y=2 \pi a+O\left(a^{2}\right), \\
E_{0}^{(2)} & =-\pi^{2} \Delta_{2}^{2}=-4 \pi^{4} a^{2}+O\left(a^{3}\right),
\end{aligned}
$$

which agrees with the result obtained using the formula of Bulla et al. [8], as it should.

To assess the quality of the perturbative estimates, we have also obtained variational bounds on the energy of the fundamental mode, using the ansatz

$$
\Psi(x, y)=\sqrt{2 \gamma} \sin (\pi y) e^{-\gamma \sqrt{\delta^{2}+x^{2}}}\left(1+\eta \frac{1-y}{1+\epsilon^{2} x^{2}}\right),
$$




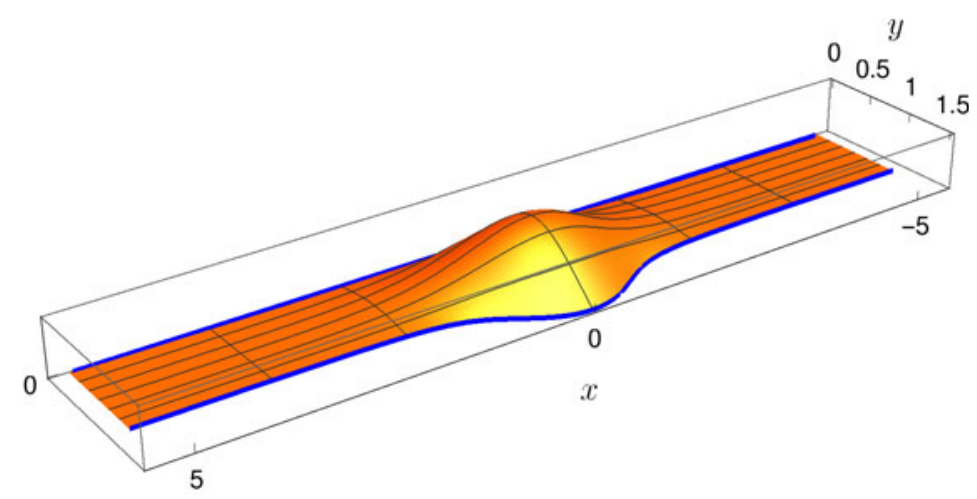

FIGURE 2. Variational wave function for the asymmetric waveguide obtained with the conformal map of equation (3.5), with $a=1 / 3$ and $z_{0}=1$.

where $\gamma, \delta, \eta$ and $\epsilon$ are variational parameters. The variational bound is

$$
E \leq E_{0}^{(\mathrm{var})}=\frac{\left\langle\Psi\left|-\nabla^{2}\right| \Psi\right\rangle}{\langle\Psi|\Sigma| \Psi\rangle} .
$$

The wave function in Figure 2 was obtained using the variational ansatz (3.6), for the case of a waveguide with $a=1 / 3$ and $z_{0}=1$, and minimizing the Rayleigh quotient in equation (3.6) with respect to the variational parameters. Note that this case is nonperturbative and the perturbative formulae cannot be applied.

Additionally, we calculate the lowest eigenvalue by a collocation (pseudospectra, discrete ordinates) method. As a check, we employ both the rational Chebyshev basis [7] and the sinh-Fourier (Cloot-Weideman) basis [3]. Both imply a domain that is a strip of uniform width, conformally mapped from the asymmetric waveguide. Conformal mapping has fallen out of favour as a grid generation scheme for numerical partial differential equation solvers because of uncontrollable and sometimes extreme nonuniformity (this is the "Geneva effect", so named because, like the diplomatic talks so frequent in that city, conformal mapping of a highly deformed region can bring distant groups together). Here, the conformal map is a small perturbation of the identity transformation and no such difficulties arise. Nonconformal coordinate changes introduce many metric factors into the transformed partial differential equation, in contrast with the single metric factor $1+\sigma(x, y)$ displayed in equation (3.2).

In Table 1, we compare the variational bounds obtained using the ansatz (3.6) with results obtained using collocation and with the second-order perturbative estimate for different values of $a$, keeping $z_{0}=1$. For $a \rightarrow 0$, the results obtained using the different methods (variational, collocation and perturbative) are very close. Note that for very small values of $a$, the wave function decays extremely slowly as $|x| \rightarrow \infty$, and the application of the collocation method becomes more challenging. 


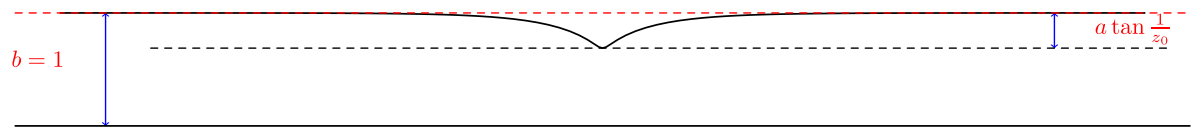

Figure 3. Asymmetric waveguide with $a<0$. The waveguide is oriented along the $x$-axis.

TABLE 1. Comparison between variational bounds, numerical results obtained using a collocation approach and perturbative corrections for the asymmetric waveguide corresponding to the map of equation (3.5) with $z_{0}=1$ for different values of $a$. Underlined digits in the third column are believed to be stable.

\begin{tabular}{llll}
\hline$a$ & $E_{0}^{(\text {var) }}-\pi^{2}$ & $E_{0}^{\text {(collocation) }}-\pi^{2}$ & $E_{0}^{(2)}$ \\
\hline $10^{-5}$ & $-3.89390 \times 10^{-8}$ & $-\underline{3.895} \times 10^{-8}$ & $-3.89636 \times 10^{-8}$ \\
$10^{-4}$ & $-3.88415 \times 10^{-6}$ & $-\underline{3.8846} \times 10^{-6}$ & $-3.89636 \times 10^{-6}$ \\
$10^{-3}$ & $-3.78211 \times 10^{-4}$ & $-\underline{3.7832} \times 10^{-4}$ & $-3.89636 \times 10^{-4}$ \\
$10^{-2}$ & $-3.03276 \times 10^{-2}$ & $-\underline{3.033396} \times 10^{-2}$ & $-3.89636 \times 10^{-2}$ \\
$10^{-1}$ & -1.08811 & $-\underline{1.0886487}$ & -3.89636 \\
\hline
\end{tabular}

3.2. Asymmetrically deformed waveguide with a localized heterogeneity We consider now the case of the waveguide discussed in Section 3.1 in the presence of a localized inhomogeneity, represented by the density

$$
\rho(u, v)=1+\rho_{0} e^{-\zeta u^{2}},
$$

where it is assumed that $\left|\rho_{0}\right| \ll 1$ and $\zeta>0$.

After applying the conformal map, we convert the original problem to an equivalent problem with density

$$
\tilde{\rho}(x, y) \approx 1+\frac{4 a\{1+\cos (2 y) \cosh (2 x)\}}{\{\cos (2 y)+\cosh (2 x)\}^{2}}+e^{-x^{2} \zeta} \rho_{0},
$$

where we assume that $|a| \approx\left|\rho_{0}\right| \ll 1$, neglecting the term depending on $a \rho_{0}$ otherwise present. In this case, one can apply the method of Amore et al. [1,2] to calculate the leading-order correction to the lowest eigenvalue using the density $\tilde{\rho}$ as

$$
\Delta_{2}=\pi \int_{-\infty}^{\infty} d x \int_{0}^{1} \tilde{\rho}(x, y) \sin ^{2} \pi y d y \approx \pi\left(2 a+\frac{\sqrt{\pi} \rho_{0}}{2 \sqrt{\zeta}}\right) .
$$

As discussed by Amore et al. [2], the condition $\Delta_{2}>0$ implies the existence of a bound state. In particular, when $a<0$ and $\Delta_{2}>0$, we consider a waveguide with a small entering deformation of the upper border and a weak inhomogeneity, which, however, is sufficient to provide binding. Interestingly, an arbitrarily small $\rho_{0}$ still provides binding, if the inhomogeneity is distributed over a sufficiently large region, that is, if $\zeta$ is sufficiently small. The case of a waveguide with $a<0$ is represented in Figure 3. Now the leading correction to the lowest eigenvalue is

$$
E_{0}^{(2)}=-\pi^{2} \Delta_{2}^{2} \approx-\pi^{4}\left(2 a+\frac{\sqrt{\pi} \rho_{0}}{2 \sqrt{\zeta}}\right)^{2} .
$$




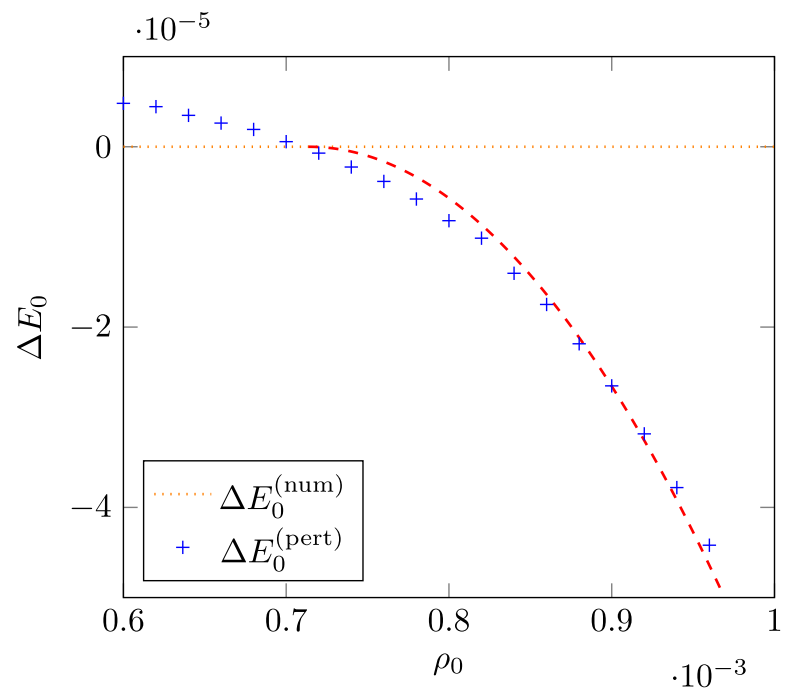

Figure 4. $\Delta E_{0}$ for an asymmetric waveguide with $a=-0.001$ and $\zeta=0.1$, as a function of $\rho_{0}$. The dashed line corresponds to the theoretical prediction of equation (3.7); the "+" symbols correspond to the numerical values obtained using collocation with mapped Chebyshev functions (a set with 101 functions along the horizontal direction and 11 along the vertical direction).

Note that the excess mass distributed on the waveguide can be calculated as

$$
\delta M=\int \rho(u, v) d u d v \approx \sqrt{\frac{\pi}{\zeta}} \rho_{0},
$$

and the energy in equation (3.7) can thus be written in a form similar to equation (3.4), as

$$
E_{0}^{(2)} \approx-\pi^{4}\left(\delta \mathcal{A}+\frac{\delta M}{2}\right)^{2} .
$$

The accuracy of this formula is demonstrated in Figure 4 for an asymmetric waveguide with $a=-0.001$ and $\zeta=0.1$, calculating the energy shift, $\Delta E_{0}=E_{0}-\pi^{2}$, as a function of $\rho_{0}$. The dashed line is the theoretical prediction of our second-order formula, whereas the "+" symbols correspond to the numerical results obtained using mapped Chebyshev functions (the scale $L=9$ has been used in all calculations and a set of 101 functions along $x$ and 11 functions along $y$ was used). Observe that the critical value of $\rho_{0}$ is quite similar in both calculations; there is, however, a mild discrepancy between the numerical and theoretical values for $\rho_{0}$ sufficiently close to the critical value. The explanation of this discrepancy is straightforward: as $\rho_{0}$ approaches the critical value, the wave function decays more and more slowly for $|x| \rightarrow \infty$; therefore, the numerical calculation should use larger sets of functions to maintain the same accuracy. Conversely, for sufficiently large $\rho_{0}$ (not shown in the figure), we also expect a discrepancy between the two curves, owing to the nonperturbative nature of the solution. 


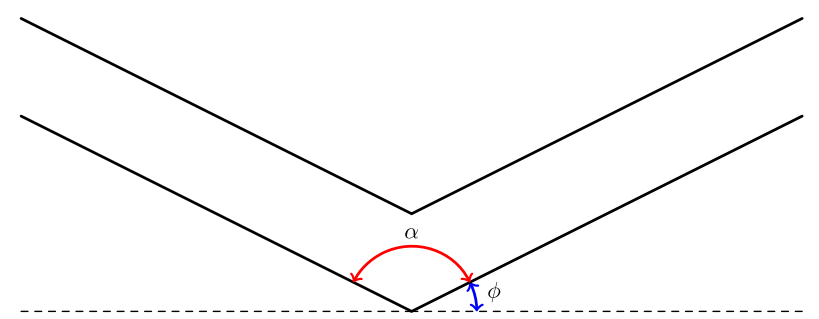

FIGURE 5. Broken strip of unit width, with the arms forming an angle $\alpha$ (the angle $\phi$ in the figure is defined by $\alpha+2 \phi=\pi)$.

3.3. Broken strip Our third example is a broken strip, that is, an infinite waveguide of constant unit width, where the two semi-infinite arms form an angle $\alpha$, as displayed in Figure 5. This problem has been studied in numerous papers [4, 5, 9-11, 14, 16, 20]. In particular, Avishai et al. [4], Duclos and Exner [11] and Granot [14] have studied the case of a weak bend, corresponding to the limit $\phi \rightarrow 0$. Avishai et al. [4] found that, in this regime, the energy of the bound state behaves as

$$
E_{0} \approx \pi^{2}-c_{\mathrm{b}} \phi^{4}
$$

with $c_{\mathrm{b}} \approx 2.10$.

The regime corresponding to sharp bends has been studied recently by Bittner et al. [5] and Sadurní and Schleich [20] using an effective potential approach, and tested experimentally using electromagnetic waveguides. This theoretical approach relies on the use of a conformal map, which transforms the broken strip into an infinite straight waveguide, and the original Helmholtz equation into a Schrödingerlike equation with an effective potential [5, equation (19)]. The conformal map considered $[5,20]$ is given by

$$
F(z)=\frac{1}{\pi} B\left(\sin ^{2}\left(\frac{\pi z}{2}\right), \frac{1}{2}-\frac{\phi}{\pi}, \frac{1}{2}+\frac{\phi}{\pi}\right),
$$

where

$$
B(x, p, q) \equiv \int_{0}^{x} u^{p-1}(1-u)^{q-1} d u
$$

is the incomplete beta function. Note that $F(z)$ maps the unit strip $x \in(0,1)$ onto a broken strip of unit width with the arms forming an angle $\alpha=\pi-2 \phi$.

Using this conformal map, we may transform the original equation into the form of equation (3.2), where

$$
\Sigma(x, y)=\left|\frac{d F}{d z}\right|^{2}=\left(1+\frac{2}{\sec (\pi x) \cosh (\pi y)-1}\right)^{2 \phi / \pi} \equiv 1+\sigma(x, y)
$$

is the "conformal density", $x \in(0,1)$ and $|y|<\infty$. Note that, in this case, the waveguide is arranged vertically and, therefore, the perturbative expressions of Amore et al. [1,2] should be adapted. 
For small bends, we may expand this density about $\phi=0$ and obtain

$$
\begin{aligned}
\Sigma(x, y) \approx & 1+\frac{2}{\pi} \phi \log \left(1+\frac{2}{\sec (\pi x) \cosh (\pi y)-1}\right) \\
& +\frac{2}{\pi^{2}} \phi^{2} \log ^{2}\left(1+\frac{2}{\sec (\pi x) \cosh (\pi y)-1}\right) \\
& +\frac{4}{3 \pi^{3}} \phi^{3} \log ^{3}\left(1+\frac{2}{\sec (\pi x) \cosh (\pi y)-1}\right)+\cdots
\end{aligned}
$$

where the density perturbation $\sigma$ can now easily be read from this expression.

Under these conditions, we are in a position to apply, directly, the approach developed by Amore et al. [1, 2]. In particular, the second-order correction to the perturbative expansion reads $E_{0}^{(2)}=-\pi^{2} \Delta_{2}^{2}$, where

$$
\begin{aligned}
\Delta_{2} & \equiv \pi \int_{0}^{1} d x \int_{-\infty}^{\infty} \sigma(x, y) \sin ^{2}(\pi x) d y \\
& =\pi \frac{2}{\pi^{2}} \phi^{2} \int_{0}^{1} d x \int_{-\infty}^{\infty} \log ^{2}\left(1+\frac{2}{\sec (\pi x) \cosh (\pi y)-1}\right) \sin ^{2}(\pi x) d y+\cdots \\
& =0.290713 \phi^{2}+O\left(\phi^{4}\right) .
\end{aligned}
$$

Note that there is no contribution of order $\phi$, since the terms in $\sigma$ corresponding to odd powers of $\phi$ are odd with respect to the change $x \rightarrow 1-x$. As a consequence of this behaviour, the second-order term in $\sigma$ in the perturbative expansion provides a leading contribution of order $\phi^{4}$ (this is consistent with the leading behaviour found by Avishai et al. [4] and Granot [14]):

$$
E_{2}=-\pi^{2} \Delta_{2}^{2} \approx-0.834119 \phi^{4} .
$$

As a result, to evaluate the dominant contribution to the energy of the fundamental mode for $\phi \rightarrow 0$, one needs to take into account the contributions of order $\phi^{4}$ arising from the third- and fourth-order perturbative expansions in $\sigma$ (the second-order contribution accounts for only about $40 \%$ of the energy).

We need to consider the third-order contribution, calculated by Amore et al. [2]

$$
E_{0}^{(3)}=-2 \pi^{2} \Delta_{2}\left(\Lambda_{1}-\Delta_{3}\right) \text {. }
$$

Using the properties of the component of $\sigma$ of order $\phi$ under the change $x \rightarrow 1-x$, we notice that

$$
\begin{aligned}
\Delta_{3} & =\pi^{3} \iint\left|y_{1}-y_{2}\right| \sigma\left(x_{1}, y_{1}\right) \sigma\left(x_{2}, y_{2}\right) \sin ^{2}\left(\pi x_{1}\right) \sin ^{2}\left(\pi x_{2}\right) d x_{1} d y_{1} d x_{2} d y_{2} \\
& =O\left(\phi^{4}\right)
\end{aligned}
$$

and, therefore, we can neglect this term in the expression for $E_{0}^{(3)}$ in equation (3.8). The remaining expression contains the integral

$$
\begin{aligned}
\Lambda_{1} \equiv \pi^{3} & \int_{-\infty}^{\infty} d y_{1} \int_{0}^{1} d x_{1} \int_{-\infty}^{\infty} d y_{2} \int_{0}^{1} \sin \left(\pi x_{1}\right) \sin \left(\pi x_{2}\right) \\
& \times \sigma\left(x_{1}, y_{1}\right) \sigma\left(x_{2}, y_{2}\right) G_{2}^{(0)}\left(x_{1}, y_{1}, x_{2}, y_{2}\right) d x_{2}
\end{aligned}
$$


TABLE 2. Asymptotic coefficient $c_{\mathrm{b}}$ estimated for $\phi=\pi / 10^{4}$ with $n_{\mathrm{b}}$ border points.

\begin{tabular}{rl}
\hline$n_{\mathrm{b}}$ & \multicolumn{1}{c}{$c_{\mathrm{b}}$} \\
\hline 100 & 2.101101624 \\
200 & 2.105792895 \\
300 & 2.10677075 \\
400 & 2.107135377 \\
500 & 2.10731157 \\
600 & 2.107410434 \\
700 & 2.107471611 \\
800 & 2.10751218 \\
900 & 2.107540509 \\
1000 & 2.107561099 \\
\hline Theoretical & 2.1077 \\
\hline
\end{tabular}

$$
\begin{aligned}
& =\pi^{3} \frac{4 \phi^{2}}{\pi^{2}} \int_{-\infty}^{\infty} d y_{1} \int_{0}^{1} d x_{1} \int_{-\infty}^{\infty} d y_{2} \int_{0}^{1} \sin \left(\pi x_{1}\right) \sin \left(\pi x_{2}\right) G_{2}^{(0, \text { odd })}\left(x_{1}, y_{1}, x_{2}, y_{2}\right) d x_{2} \\
& \quad \times \log \left(1+\frac{2}{\sec \left(\pi x_{1}\right) \cosh \left(\pi y_{1}\right)-1}\right) \log \left(1+\frac{2}{\sec \left(\pi x_{2}\right) \cosh \left(\pi y_{2}\right)-1}\right)+O\left(\phi^{3}\right) \\
& \approx 0.171407 \phi^{2}
\end{aligned}
$$

and the third-order correction to the energy

$$
E_{3}=-2 \pi^{2} \Delta_{2} \Lambda_{1}+O\left(\phi^{5}\right) \approx-0.983607 \phi^{4},
$$

providing approximately $47 \%$ to the leading-order correction.

The calculation of the fourth-order contribution involves selecting the contributions of order $\phi^{4}$ for use in the expression for $E_{0}^{(4)}$, using the explicit expressions for $\Delta_{i}$ and $\Lambda_{i}$ provided. Simple inspection proves that there is only one term contributing to the leading order, and the energy reduces to

$$
E_{4}=-\pi^{2} \Lambda_{1}^{2} \approx-0.289971 \phi^{4}
$$

corresponding to roughly $13 \%$ of the total correction. When we combine the three contributions

$$
\Delta E \approx-\pi^{2}\left[\Delta_{2}+\Lambda_{1}\right]^{2} \approx-2.1077 \phi^{4},
$$

which is extremely close to the value of $c_{\mathrm{b}}$ estimated by Avishai et al. [4].

In Table 2, we compare this theoretical value with the values obtained numerically, following the approach of Granot [14] for $\phi=\pi / 10^{4}$, using an increasing number of points $n_{\mathrm{b}}$ at which the continuity of the solution is imposed.

In Figure 6 we plot the values in the table, and compare the asymptotic coefficient obtained from the best fit of these values with the theoretical values obtained from 


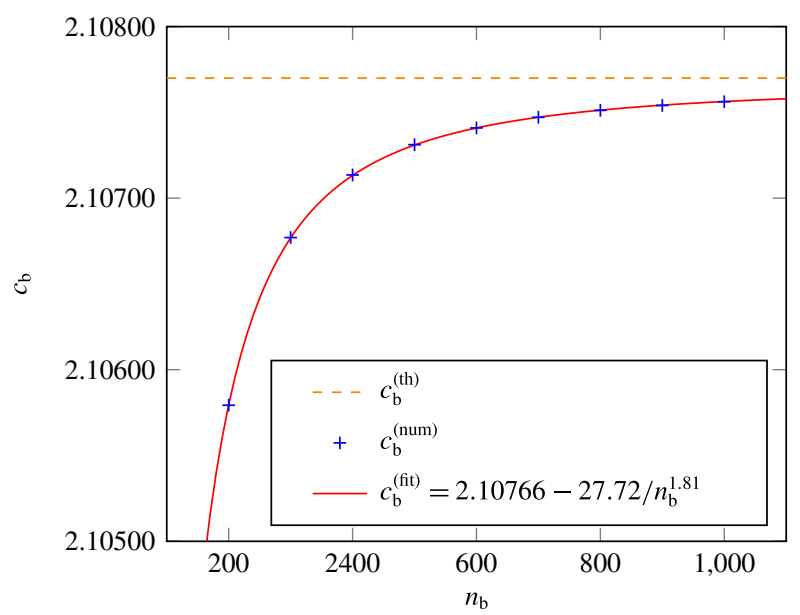

FIGURE 6. Comparison between numerical and theoretical estimates of $c_{\mathrm{b}}$.

the explicit formulae. The best fit of the numerical values shows an excellent agreement with the theoretical value calculated using the perturbative formulae of Amore et al. [1, 2].

\section{Conclusions}

We have considered three examples of infinite waveguides, where the exact perturbative formulae of Amore et al. [1, 2] apply. In particular, for the cases of an infinite homogeneous and asymmetric waveguide and a broken strip, our results agree with the analogous results obtained by applying a formula derived by Bulla et al. [8] for an asymmetric waveguide and with the numerical results obtained by Avishai et al. [4] and Granot [14] for a broken strip. Note that the formula of Bulla et al. [8] is limited to the case of asymmetric homogeneous waveguides, whereas the formulae of Amore et al. [1,2] apply to more general geometries (the broken strip is just one example), even in the presence of heterogeneities. This latter case has been studied in the second example, an asymmetric heterogeneous waveguide, demonstrating that the theoretical results obtained using the formulae of Amore et al. [2] are in perfect agreement with the numerical results obtained using a collocation scheme. To the best of our knowledge, Amore et al. [1,2] provide the only calculation in which the effect of both deformations and heterogeneity is derived; this paper provides a numerical verification of those formulae.

An important problem related to the problem considered in this paper is the study of the transport problem in waveguides that are weakly deformed or contain small heterogeneities (or both). The study of this problem, however, cannot be accomplished with the method of Gat and Rosenstein [13], which only works when the reference state belongs to the discrete part of the spectrum. 


\section{Acknowledgements}

The research of P. Amore and P. Zhevandrov was supported by the Sistema Nacional de Investigadores (México). J. P. Boyd was supported by the National Science Foundation of the US under DMS-1521158. The figures were produced using TikZ [22].

\section{References}

[1] P. Amore, "Weakly bound states in heterogeneous waveguides: a calculation to fourth order". Preprint, 2016, arXiv:1601.02470.

[2] P. Amore, F. M. Fernández and C. P. Hofmann, "Weakly bound states in heterogeneous waveguides", Eur. Phys. J. B 89 (2016) 163; doi:10.1140/epjb/e2016-70197-0.

[3] J. A. C. Weideman and A. Cloot, "Spectral methods and mappings for evolution equations on the infinite line", Comput. Methods Appl. Mech. Eng. 80 (1990) 467-481; doi:10.1016/0045-7825(90)90052-N.

[4] Y. Avishai, D. Bessis, B. G. Giraud and G. Mantica, "Quantum bound states in open geometries", Phys. Rev. B 44 (1991) 8028-8034; doi:10.1103/PhysRevB.44.8028.

[5] S. Bittner, B. Dietz, M. Miski-Oglu, A. Richter, C. Ripp, E. Sadurní and W. P. Schleich, "Bound states in sharply bent waveguides: analytical and experimental approach", Phys. Rev. E 87 (2013) 042912; doi:10.1103/PhysRevE.87.042912.

[6] D. Borisov, P. Exner, R. Gadyl'shin and D. Krejčiřik, "Bound states in weakly deformed strips and layers", Ann. Henri Poincaré 2 (2001) 553-572; doi:10.1007/PL00001045.

[7] J. P. Boyd, "Spectral methods using rational basis functions on an infinite interval", J. Comput. Phys. 69 (1987) 112-142; doi:10.1016/0021-9991(87)90158-6.

[8] W. Bulla, F. Gesztesy, W. Renger and B. Simon, "Weakly coupled bound states in quantum waveguides", Proc. Amer. Math. Soc. 125 (1997) 1487-1495; doi:10.1090/S0002-9939-97-03726-X.

[9] J. P. Carini, J. T. Londergan, K. Mullen and D. P. Murdock, "Bound states and resonances in waveguides and quantum wires", Phys. Rev. B 46 (1992) 15538; doi:10.1103/PhysRevB.46.15538.

[10] J. P. Carini, J. T. Londergan, K. Mullen and D. P. Murdock, "Multiple bound states in sharply bent waveguide", Phys. Rev. B 48 (1993) 4503-4515; doi:10.1103/PhysRevB.48.4503.

[11] P. Duclos and P. Exner, "Curvature-induced bound states in quantum waveguides in two and three dimensions", Rev. Math. Phys. 7 (1995) 73-102; doi:10.1142/S0129055X95000062.

[12] P. Exner and H. Kovařík, Quantum waveguides (Springer, Cham, 2015); doi:10.1007/978-3-319-18576-7.

[13] G. Gat and B. Rosenstein, "New method for calculating binding energies in quantum mechanics and quantum field theories", Phys. Rev. Lett. 70 (1993) 5-8; doi:10.1103/PhysRevLett.70.5.

[14] E. Granot, "Emergence of a confined state in a weakly bent wire", Phys. Rev. B 65 (2002) 233101; doi:10.1103/PhysRevB.65.233101.

[15] N. E. Hurt, Mathematical physics of quantum wires and devices: from spectral resonances to Anderson localization (Kluwer, Dordrecht, 2000); doi:10.1007/978-94-015-9626-8.

[16] D. Levin, "On the spectrum of the Dirichlet Laplacian on broken strips", J. Phys. A 37 (2003) L9-L11; doi:10.1088/0305-4470/37/1/L02.

[17] J. T. Londergan, J. P. Carini and D. P. Murdock, Binding and scattering in two-dimensional systems: applications to quantum wires, waveguides and photonic crystals (Springer, Berlin, 1999); doi:10.1007/3-540-47937-6.

[18] S. A. Nazarov, "Discrete spectrum of cranked, branching, and periodic waveguides", St. Petersburg Math. J. 23 (2012) 351-379; doi:10.1090/S1061-0022-2012-01200-8.

[19] M. Reed and B. Simon, Methods of modern mathematical physics. IV. Analysis of operators (Academic Press, New York, 1978). 
[20] E. Sadurní and W. P. Schleich, "Conformal mapping and bound states in bent waveguides", AIP Conf. Proc. 1323 (2010) 283; doi:10.1063/1.3537857.

[21] B. Simon, "The bound state of weakly coupled Schrödinger operators in one and two dimensions", Ann. Phys. 97 (1976) 279-288; doi:10.1016/0003-4916(76)90038-5.

[22] T. Tantau, "The TikZ and PGF Packages, Manual for version 3.0.0"; http://sourceforge.net/projects/pgf/. 